\title{
Magnetic Resonance Imaging of the Brain without Contrast
}

National Cancer Institute

\section{Source}

National Cancer Institute. Magnetic Resonance Imaging of the Brain without Contrast. NCI Thesaurus. Code C137874.

Magnetic resonance imaging of the brain without the use of a contrast agent. 